\title{
Preface \\ High Performance Computing at the Poznan Supercomputing and Networking Center in 2011
}

This is already the second time we have published the results of the annual work of our HPC (High Performance Computing) users. The current issue presents the results from 2011. Poznan Supercomputing and Networking Center has been providing computing power continuously since 1994. Since that time, the nature and way of sharing computing power has changed significantly. In addition to traditional grant applications that give users access to HPC resources in the country, there are also virtual organizations (VOs), for which PSNC made available its resources. The first virtual organizations appeared in 2007 within the framework of European projects: EGEE (Enabling Grids for E-sciencE, http://www.eu-egee.org/) and WLCG (The Worldwide LHC Computing Grid, http://wlcg . web.cern.ch /).

In 2011, virtual organizations used 7 million hours of CPU time (in comparison to 0.5 million hours of CPU in 2007) allocated by the queuing system, the record holder is ALICESGM (A Large Ion Collider Experiment At CERN LHC). HPC users have used 9 million CPU hours. The total of all users used 16 million hours allocated by the queuing system, and is an increase of $100 \%$ (in comparison to 2010) of the CPU time that was dedicated to HPC users.

The computing and data Infrastructures of PSNC are nowadays an inseparable part of the European and national infrastructure. Among the major infrastructure projects that provide resources to the scientific community of Polish and foreign are:

- Platon - "PLATON - Science Services Platform" (http://www.platon.pionier.net.pl/)

- PL-GRID Plus - Domain-oriented services and resources of Polish Infrastructure for Supporting Computational Science in the European Research Space (http://www.plgrid.pl/en)

- POWIEW - HPC Infrastructure for Grand Challenges of Science and Engineering (http://wielkiewyzwania.pl/)

- KMD2 - National Data Storage (http://kmd.pcss.pl/)

- PRACE -1IP - PRACE - FIRST Implementation Phase Project (http://www.prace-ri.eu/)

- PRACE-2IP - PRACE - 2nd Implementation Phase Project;

- EGI-InSPIRE - Integrated Sustainable Pan-European Infrastructure for Researchers in Europe (http://www.egi.eu/about/egi-inspire/);

- WLCG - Worldwide LHC Computing Grid (http://wlcg.web.cern.ch/)

- EUDAT - European Data Infrastructure (http://www.eudat.eu/).

PRACE projects are also worth mentioning. They give the Polish scientific community access to the largest HPC resources in Europe, which are on the list of Top 500 (www.top500.org), with a capacity exceeding Ptflops.

In the near future the same will happen to large archivers for data that will be made available in the European space data infrastructure. Meanwhile, our national activity is ahead of the European one. Examples are national structural projects, including

- PLATON - dedicates a large space for data storage (archiving service) and cloud computing (campus computing), national wide and especially among the 21 largest university campuses belonging to PIONIER consortium.

- PL-Grid - dedicates grid resources in 5 HPC centres (Kraków, Gdańsk, Wrocław, Warszawa, Poznań) for scientific communities

- POWIEW - dedicates advanced HPC resources demanded by grand challenges.

There are 88 computing grants in PSNC with 660+ registered users in 2011. Two centers of excellence were also established as a joint initiative of the Institute of Bioorganic Chemistry of the Polish Academy of Sciences, and the Institute of Radio Astronomy, Nicolaus Copernicus University in Torun, Poland:

- 3D RNA structure prediction and visualisation

- Competence Center Computational Astrophysics.

It is our pleasure to present you some selected papers on: human genetics, physical chemistry, organic chemistry and bioorganic and mechanical engineering. They describe some of the results achieved last year and based on PSNC computing resources. 